\title{
Algorithm for Automatic Analysis of Smooth Pursuit Eye Movements Using a Combination of Video- oculography and Deep Learning-Based Object Detection
}

\author{
Masakazu Hirota ( $\nabla$ hirota.ortho@med.teikyo-u.ac.jp ) \\ Teikyo University \\ Kanako Kato \\ Teikyo University \\ Megumi Fukushima \\ Teikyo University \\ Yuka Ikeda \\ Teikyo University \\ Takao Hayashi \\ Teikyo University \\ Atsushi Mizota \\ Teikyo University
}

\section{Research Article}

Keywords:

Posted Date: February 9th, 2022

DOI: https://doi.org/10.21203/rs.3.rs-1301293/v1

License: (a) (i) This work is licensed under a Creative Commons Attribution 4.0 International License.

Read Full License 


\section{Abstract}

In the evaluation of smooth pursuit eye movements (SPEMs), recording the stimulus onset time is mandatory. In the laboratory, the stimulus onset time is recorded by electrical signal or programming, and video-oculography (VOG) and the visual stimulus are synchronized. Nevertheless, because the examiner must manually move the fixation target, recording the stimulus onset time is challenging in daily clinical practice. Thus, this study aimed to develop an algorithm for evaluating SPEMs while testing the ninedirection eye movements without recording the stimulus onset time using VOG and deep learning-based object detection (single-shot multibox detector), which can predict the location and types of objects in a single image. The algorithm of peak fitting-based detection correctly classified the directions of target orientation and calculated the latencies and gains within the normal range while testing the nine-direction eye movements in healthy individuals. These findings suggest that the algorithm of peak fitting-based detection has sufficient accuracy for the automatic evaluation of SPEM in clinical settings.

\section{Introduction}

Eye movements include the ability to fixate and track visual stimuli. In most ophthalmology clinics, the examiner evaluates smooth pursuit eye movements (SPEMs) by subjectively noting their accuracy in relation to a target that is being moved manually to nine directions by the examiner. At the same time, the patient follows it with his or her eyes. ${ }^{1-8}$ Nevertheless, because the laboratory methods for quantifying eye movements are constrained by the presentation of the target, no objective evaluation method has been established for use in daily clinical practice..$^{9-11}$

To achieve a high accuracy in eye movement testing, it is necessary to present a predetermined target on the liquid crystal monitor according to the programming codes. Nevertheless, presenting targets according to the prescribed protocol is difficult in the clinical setting, because the examiner must modify the movement of the target as appropriate to examine the suspected abnormality. ${ }^{12-16}$ In their approach to obtaining an accuracy of eye movement measurement in the clinical field that is close to the laboratory level, Hirota et al. ${ }^{17}$ reported that a single-shot multibox detector (SSD) ${ }^{18}$ which is an algorithm for deep learning-based object detection, achieved high accuracy in recognizing the target that was moved manually, and the target location was significantly and highly positively correlated with the positions of both eyes, as recorded by the video-oculography (VOG; the VOG-SSD system). Moreover, the processing speed was faster in the algorithm for deep learning-based object detection than in the simpler conventional algorithms that use raster scans per image. ${ }^{19}$ Although a previous study reported that the target location and both eye positions could be recorded simultaneously in real space, this has not been implemented within an automatic eye movement analysis, such as latency and gain.

Recording the stimulus onset time is mandatory when evaluating eye movements. In the laboratory, the stimulus onset time is recorded by the electrical signal, and the VOG and the visual stimulus are synchronized. Nevertheless, because the examiner must manually move the fixation target, recording the stimulus onset time is challenging in daily clinical practice. Thus, this study aimed to develop an 
algorithm for evaluating SPEM in nine-gaze direction testing using the VOG-SSD system without recording the stimulus onset time.

\section{Methods}

\section{General procedures}

\subsection{Subjects}

A total of 21 young adult volunteers (age [mean \pm standard deviation], $21.4 \pm 1.4$ years] participated in this study. All subjects underwent complete ophthalmologic examinations, including determination of the ocular dominance using the hole-in-the-card test, best-corrected visual acuity at a distance $(5.0 \mathrm{~m})$, near the point of convergence, stereoscopic acuity at $40 \mathrm{~cm}$ (Titmus Stereotest; Stereo Optical Co., Inc., Chicago, IL, USA), heterophoria by the alternating cover test at near $(33 \mathrm{~cm})$ and at distance $(5.0 \mathrm{~m})$ assessments, and fundus examinations. Stereoacuity was converted into the logarithm of the arc second (log arcsec).

Table 1 presents the characteristics of the subjects. The mean \pm standard deviation of the refractive errors (spherical equivalents) of the dominant eye was $-3.23 \pm 3.00 \mathrm{D}$ and that of the nondominant eye was $-3.08 \pm 2.80 \mathrm{D}$. The best-corrected visual acuity was 0.0 logMAR units or better in all subjects. The average heterophoria was $-6.3 \pm 5.9$ prism diopters (PDs) at distance and $-10.9 \pm 8.8$ PDs at near. All healthy volunteers had a stereoacuity of $1.62 \pm 0.05 \mathrm{log} \operatorname{arcsec}$ (range, 40-60 s).

After we explained the nature of the study and possible complications to the subjects, all subjects provided informed consent. This investigation adhered to the World Medical Association Declaration of Helsinki tenets. The Institutional Review Board of Teikyo University approved the experimental protocol and consent procedures (approval No. 19-224-2).

\subsection{Apparatus}

In this study, we used the VOG-SSD system developed by Hirota et al.. ${ }^{17}$ We recorded eye movements while tracking the target using a VOG (EMR-9, NAC Image Technology Inc., Tokyo, Japan). The VOG device determined the eye positions by detecting the corneal reflex and pupil center that were created by the reflection of near-infrared light with a sampling rate of $240 \mathrm{~Hz}$. The measurement error (interquartile range) was $0.2^{\circ}-0.5^{\circ}$ at a distance of $1.0 \mathrm{~m}$. The scene camera recorded the real scenes (resolution, 640 $\times 480$ pixels; angle of view, $\pm 31^{\circ}$ from the center of the scene camera) with a sampling rate of $29.97 \mathrm{~Hz}$. The gaze positions were merged with the real scenes at a delay of $\leq 52 \mathrm{~ms}$.

Before performing the eye movement test, all subjects underwent a calibration test to adjust the position of their gaze on the images of the scene camera and under binocular conditions with fully corrected glasses. All subjects were asked to fixate nine red cross targets (visual angle, $0.1^{\circ}$ ) on a white calibration plate during calibration. From one to nine, the nine red crosses of the targets were set at the following 
parameters: (center: horizontal of $0.0^{\circ}$, vertical of $0.0^{\circ}$ ), (left: $-20.0^{\circ}, 0.0^{\circ}$ ), (right: $0.0^{\circ}, 20.0^{\circ}$ ), (upper left: $-20.0^{\circ}, 20.0^{\circ}$ ), (upper right: $\left.20.0^{\circ}, 20.0^{\circ}\right)$, (lower left: $\left.-20.0^{\circ},-20.0^{\circ}\right)$, (lower right: $20.0^{\circ},-20.0^{\circ}$ ), (upper: $20.0^{\circ}, 0.0^{\circ}$ ), and (lower: $0.0^{\circ},-20.0^{\circ}$ ) respectively. The center of the calibration plate was defined as $0^{\circ}$, the right and upper halves of the screen were defined as the positive sides, and the left and lower halves were defined as the negative sides.

The object detection algorithm was used for the SSD model that was the same as in Hirota et al. ${ }^{17,18}$, which detected the target of rabbit-like character with a $75 \%$ average precision of $99.7 \% \pm 0.6 \%$.

We used Python 3.8.5 for Windows 10 (Microsoft, Redmond, WA, USA) with the following libraries: Matplotlib 3.3.2, Numpy 1.18.5, OpenCV 3.3.1, Pandas 1.1.3, Pytorch 1.6.0, Scikit-learn 0.23.2, and Seaborn 0.11.0.

\subsection{Nine-direction eye movements testing}

The target was a rabbit-like character that had already been learned to the SSD in Hirota et al.. ${ }^{17}$ The target size was $10 \times 10 \mathrm{~cm}$, which subtended a visual angle of $5.7^{\circ}$ at $1.0 \mathrm{~m}$. The target was manually moved to nine directions (center, left, right, upper left, upper right, lower left, lower right, upper, and lower) within $\pm 15^{\circ}$ randomly by an examiner.

All subjects were seated in a well-lit room (600 lx) wearing fully corrective spectacles. Each subject's head was stabilized with a chin rest and forehead rest. During the eye movement test, the subjects were asked to fixate on the nose of the target, the visual angle of which was $0.1^{\circ}$ at $1.0 \mathrm{~m}$.

\subsection{Filtering for both eye positions}

We excluded VOG data when the change in pupil diameter was $>2 \mathrm{~mm} /$ frame due to blinking. ${ }^{20} \mathrm{We}$ replaced the percentage of missing values $(0.4 \% \pm 0.7 \%$ for all subjects) with a linearly interpolated value calculated from an algorithm written with Python 3.8.5. The horizontal and vertical eye movements were analyzed, and the SPEM and saccadic eye movements were identified using a velocity-threshold identification (I-VT) filter. ${ }^{21}$ The I-VT filter was used to classify eye movements on the basis of the velocity of the directional shifts of the eye. A saccadic eye movement was defined as the median velocity of three consecutive windows $>100 \%$ s. Then, the eye position data at $240 \mathrm{~Hz}$ were synchronized with the target data at $29.97 \mathrm{~Hz}$.

\section{Experiment 1}

Eye movement testing involves moving the target in eight directions: left, right, upper left, upper right, lower left, lower right, upper, and lower. There is a need for an algorithm that can identify the direction in which the examiner moves the target manually in the clinic without the input of a trigger. In experiment 1 , we compared the accuracy of the classification in each direction of target presentation between the peak fitting-based detection algorithm and the conventional threshold-based detection algorithm. 


\subsection{Procedures}

In clinical practice, the origin of the scene camera (horizontal of $0.0^{\circ}$, vertical of $0.0^{\circ}$ ) and the position where the target is initially presented by the examiner do not necessarily coincide (Fig. 1A, B). The median of the target location of the target was calculated both horizontally and vertically, respectively, and was defined as the relative origin. The target location and both eye positions were corrected for the difference from the relative origin (Fig. 1C).

The target location calculated using the SSD was identified more than $99 \%$ of the time and was more stable than eye positions affected by blinks and tears. Thus, each direction was identified using the location of the target as a cue.

\subsection{Algorithm of automatic detection for testing the directions of eye movements}

\subsubsection{Peak fitting-based detection}

The target location was converted to the position vector, and then, the maximum and minimum peaks were detected for $3.0 \mathrm{~s}$ (Fig. 2A, B). We separated the data between the two minimum peaks, including one maximum peak. The separated data were decomposed into horizontal and vertical components from the position vector (Fig. 2C, D). After excluding $1 \mathrm{~s}$ from both ends of the separated data, the medians of the horizontal and vertical target locations were calculated (Fig. 2E, F).

The eight median horizontal and vertical locations were ranked from maximum to minimum at left, right, upper, and lower, and then the top three values in four directions were grouped (Fig. 3A). The upper left, upper right, lower left, and lower right were identified by combining the horizontal and vertical directions (Fig. 3B). The remaining data in each group were the left, right, upper, and lower.

\subsubsection{Threshold-based detection}

Threshold-based detection is a simple approach for identifying the category. In this study, the target data were separated to left (horizontal location $\leq-2.0^{\circ}$ and $-2.0^{\circ} \leq$ vertical location $\left.\leq+2.0^{\circ}\right)$, right $(+2.0 \leq$ horizontal location and $-2.0^{\circ} \leq$ vertical location $\leq+2.0^{\circ}$ ), upper left (horizontal location $\leq-2.0^{\circ}$ and $+2.0^{\circ} \leq$ vertical location), upper right $\left(+2.0 \leq\right.$ horizontal location and $+2.0^{\circ} \leq$ vertical location), lower left (horizontal location $\leq-2.0^{\circ}$ and vertical location $\left.\leq-2.0^{\circ}\right)$, lower right $(+2.0 \leq$ horizontal location and vertical location $\left.\leq-2.0^{\circ}\right)$, upper $\left(-2.0 \leq\right.$ horizontal location $\leq+2.0^{\circ}$ and $+2.0^{\circ} \leq$ vertical location $)$, and lower $\left(-2.0 \leq\right.$ horizontal location $\leq+2.0^{\circ}$ and vertical location $\left.\leq-2.0^{\circ}\right)$. The cutoff value was defined as the minimum value calculated using the averaged degree of the mean -2.0 standard deviation in all directions using the data from subject 1 to subject 5 .

\subsection{Statistical analysis}

We evaluated the accuracy of the classification in each direction between the peak fitting-based and threshold-based detection using Fisher's exact test. 
SPSS version 26 (IBM Corp., Armonk, NY, USA) was used to determine the significance of the differences, and a $P$ value of $<0.05$ was considered to be statistically significant.

\subsection{Results}

The accuracy of the classification in each direction was significantly higher using the peak fitting-based detection (correct, $100.0 \%$; incorrect, $0.0 \%$ ) than using the threshold-based detection (correct, $47.8 \%$; incorrect, 52.2\%; $P<0.001$; Table 2).

The finding of experiment 1 suggested that the algorithm of the peak fitting-based detection was suitable for evaluating eye movement testing.

\section{Experiment 2}

In experiment 2, we investigated the algorithm for the automatic calculation of latency and gain, which are evaluation indices of the eye movements using the data obtained by the peak fitting-based detection algorithm.

\subsection{Calculating for latency and gain}

All directions of the horizontal and vertical target location and both eye positions were converted to the position vector. The raw data were fitted with a cubic function and were detected at each peak time (Fig. 4A, B). Then, each peak time was applied to the raw data (Fig. 4C). The latencies of both eyes were defined as the difference between the peak time in both eyes and that in the target location.

The target location and both eye positions at the peak time were defined as maximum values. We explored the 25th and 75th percentile points of the maximum values in the centrifugal direction (Fig. 5). We then created a linear regression line using the target location and both eye positions between the 25th and 75th percentile points of the maximum values. The gains of both eyes were defined as the ratio of the slope of the regression line in both eyes to the slope of the regression line in the target between the 25th and 75th percentile points.

\subsection{Statistical analysis}

We determined the differences in the latencies and gains within both eyes in each direction using the Schéffe test. We calculated the differences in the latencies and gains between both eyes in each direction using the Wilcoxon signed-rank test. The Bonferroni method was used to adjust the $P$ values.

To determine the significance of the differences, we used SPSS version 26 (IBM Corp., Armonk, NY, USA), and a $P$ value of $<0.05$ was considered to be statistically significant.

\subsection{Results}


The latencies in all directions were not significantly different within both eyes (left eye, $P>0.150$; right eye, $P>0.68$; Fig. 6A, B; Table 3$)$. The latencies in all directions were not significantly different between left (138.04 $\pm 89.36 \mathrm{~ms}$ in all directions) and right (144.75 $\pm 97.78 \mathrm{~ms}$ in all directions) eyes $(P>0.552$; Fig. 6C; Table 3).

The gains in all directions were not significantly different within both eyes (left eye, $P>0.75$; right eye, $P>$ $0.50 ;$ Fig. 7A, B; Table 3). The gains in all directions were not significantly different between left ( $0.943 \pm$ 0.149 in all directions) and right ( $0.935 \pm 0.133$ in all directions) eyes $(P>0.99 ;$ Fig. $7 \mathrm{C}$; Table 4$)$.

The findings of experiment 2 suggest that using the algorithm of peak fitting-based detection, the eye movements can be evaluated from the data with the identified target direction.

\section{Discussion}

Recording the stimulus onset time is mandatory when evaluating eye movements. Nevertheless, because the examiner must move the fixation target manually, recording the stimulus onset time is challenging in daily clinical practice. In this study, we developed an algorithm of peak fitting-based detection to evaluate SPEM in nine-direction testing without recording the stimulus onset time. We found that the present algorithm had high accuracy for identifying the directions of target orientation in nine-direction testing.

The peak fitting-based detection algorithm correctly classified all directions of target orientation. We consider that the present algorithm is advantageous for identifying the oblique directions because it calculates the top three in the left, right, upper, and lower directions and detects the oblique direction by combining the two directions, resulting in high accuracy. By contrast, the classification accuracy of the threshold-based detection algorithm was about half that of the peak fitting-based detection algorithm. Additionally, there are no standard criteria for determining the threshold, although in this study, the threshold was determined from the standard deviation of five subjects. Since the standard deviation contains $68 \%$ of the total data, the decrease of the classification accuracy of the threshold-based detection algorithm to chance level indicates that the influence of oblique direction is quite significant in the automatic determination of nine-direction testing.

The latencies (mean latencies of left and right eyes, 138.04 and $144.75 \mathrm{~ms}$, respectively) and gains (mean gains of left and right eyes, 0.943 and 0.935 , respectively), which we calculated from the data of the identified target direction using the algorithm of peak fitting-based detection, were similar to those reported in earlier studies: the latency of the SPEM was between 50 and $300 \mathrm{~ms}^{22-24}$ the gain of SPEM was greater than 0.90 under a velocity of $10^{\circ} \%$, and the moving distance was $15^{\circ}$ in healthy individuals. ${ }^{25}$ These results suggest that the automatic method for calculating latency and gain which we implemented in this study may has a slight error compared to the manual analysis because of the fitting with cubic functions to calculate the vertices, however, the error is acceptable. 
This study has a limitation. The method for identifying the top three combinations in the horizontal and vertical directions had high classification accuracy. However, when the same direction is examined twice, the accuracy might decrease due to the overflow in one direction. Thus, to overcome these limitations, we plan to use this algorithm to accumulate data and use machine learning to identify the target direction in future work.

\section{Conclusion}

The algorithm of peak fitting-based detection correctly classified the directions of the target orientation and calculated the latencies and gains within the normal range during nine-direction eye movement testing in healthy individuals. These findings suggest that the peak fitting-based detection algorithm has an accuracy that is sufficient for the automatic evaluation of SPEM in clinical settings.

\section{Declarations}

\section{Acknowledgments}

This work was supported by Grands-in-Aid for Early-Career Scientists, Scientific Research (A) and (B), Challenging Exploratory Research, Japan Society for the Promotion of Science (19K20728 [M.H.], $18 \mathrm{H} 04116$ [M.H.], $20 \mathrm{~K} 04271$ [M.H.], and 19K21783 [Y.I. and M.H.], respectively); Charitable Trust Fund for Ophthalmic Research in Commemoration of Santen Pharmaceutical's Founder (M.H.); Takeda Science Foundation (M.H.); Nakatani Foundation for Advancement of Measuring Technology in Biomedical Engineering (M.H.); Japan Medical Herb Association Research Grant Program (M.H.).

\section{Author contributions}

M.H. conceived the project and designed the experiments. M.H. produced the apparatus. M.H., K.K., and M.F. performed experiments. M.H., K.K., Y.I., T.H., and A.M. analyzed the data. M.H. and A.M. wrote the manuscript. All authors reviewed the manuscript.

\section{Competing interests.}

M.H., The Patent no. is 2021-110320 (P); K.K., None; M.F., None; Y.I., None; T.H., None; A.M., None.

\section{Data Availability}

The data that support the findings of this study are openly available in Notion at https://www.notion.so/Algorithm-for-Automatic-Analysis-of-Smooth-Pursuit-Eye-Movements-Using-aCombination-of-Video-oculog-

af5cafa832f04c7297022795193cbd48\#5ead35fc5352448098afb470aff41c38

\section{References}


1. Rashbass, C. The relationship between saccadic and smooth tracking eye movements. J. Physiol. 159, 326-338, doi:10.1113/jphysiol.1961.sp006811 (1961).

2. Robinson, D. A. The mechanics of human smooth pursuit eye movement. J. Physiol. 180, 569-591 (1965).

3. Westheimer, G. \& McKee, S. P. Visual acuity in the presence of retinal-image motion. J. Opt. Soc. Am. 65, 847-850, doi:10.1364/josa.65.000847 (1975).

4. Kowler, E., van der Steen, J., Tamminga, E. P. \& Collewijn, H. Voluntary selection of the target for smooth eye movement in the presence of superimposed, full-field stationary and moving stimuli. Vision Res. 24, 1789-1798, doi:10.1016/0042-6989(84)90010-5 (1984).

5. Lisberger, S. G., Morris, E. J. \& Tychsen, L. Visual motion processing and sensory-motor integration for smooth pursuit eye movements. Annu. Rev. Neurosci. 10, 97-129, doi:10.1146/annurev.ne.10.030187.000525 (1987).

6. Krauzlis, R. J. Recasting the smooth pursuit eye movement system. J. Neurophysiol. 91, 591-603, doi:10.1152/jn.00801.2003 (2004).

7. Heinen, S. J., Potapchuk, E. \& Watamaniuk, S. N. A foveal target increases catch-up saccade frequency during smooth pursuit. J. Neurophysiol. 115, 1220-1227, doi:10.1152/jn.00774.2015 (2016).

8. Shanidze, N., Ghahghaei, S. \& Verghese, P. Accuracy of eye position for saccades and smooth pursuit. J Vis 16, 23, doi:10.1167/16.15.23 (2016).

9. Levin, S. et al. Smooth pursuit eye movements in schizophrenics: quantitative measurements with the search-coil technique. J. Psychiatr. Res. 22, 195-206, doi:10.1016/0022-3956(88)90005-2 (1988).

10. Imai, T. et al. Comparing the accuracy of video-oculography and the scleral search coil system in human eye movement analysis. Auris Nasus Larynx 32, 3-9, doi:10.1016/j.anl.2004.11.009 (2005).

11. Ingster-Moati, I. et al. Vertical and horizontal smooth pursuit eye movements in children: A neurodevelopmental study. Eur. J. Paediatr. Neurol. 13, 362-366, doi:10.1016/j.ejpn.2008.07.003 (2009).

12. Harley, R. D. Paralytic strabismus in children. Etiologic incidence and management of the third, fourth, and sixth nerve palsies. Ophthalmology 87, 24-43, doi:10.1016/s0161-6420(80)35280-9 (1980).

13. Fukushima, J., Tanaka, S., Williams, J. D. \& Fukushima, K. Voluntary control of saccadic and smoothpursuit eye movements in children with learning disorders. Brain Dev. 27, 579-588, doi:10.1016/j.braindev.2005.03.005 (2005).

14. Lions, C., Bui-Quoc, E., Wiener-Vacher, S., Seassau, M. \& Bucci, M. P. Smooth pursuit eye movements in children with strabismus and in children with vergence deficits. PLoS One 8, e83972, doi:10.1371/journal.pone.0083972 (2013).

15. Metsing, I. \& Ferreira, J. The prevalence of poor ocular motilities in a mainstream school compared to two learning-disabled schools in Johannesburg. African Vision and Eye Health 75, a328, doi:http://dx.doi.org/10.4102/aveh.v75i1.328 (2015). 
16. Raashid, R. A., Liu, I. Z., Blakeman, A., Goltz, H. C. \& Wong, A. M. The Initiation of Smooth Pursuit is Delayed in Anisometropic Amblyopia. Invest. Ophthalmol. Vis. Sci. 57, 1757-1764, doi:10.1167/iovs.16-19126 (2016).

17. Hirota, M., Hayashi, T., Watanabe, E., Inoue, Y. \& Mizota, A. Automatic Recording of the Target Location During Smooth Pursuit Eye Movement Testing Using Video-Oculography and Deep Learning-Based Object Detection. Translational Vision Science \& Technology 10, 1, doi:10.1167/tvst.10.6.1 (2021).

18. Liu, W. et al. SSD: Single Shot MultiBox Detector. arXiv e-prints, arXiv:1512.02325 (2015). <https://ui.adsabs.harvard.edu/abs/2015arXiv151202325L>.

19. Cho, H., Rybski, P. E., Bar-Hillel, A. \& Zhang, W. Real-time pedestrian detection with deformable part models. 2012 IEEE Intelligent Vehicles Symposium 2012, 1035-1042, doi:10.1109/IVS.2012.6232264. (2012).

20. Kwon, K. A. et al. High-speed camera characterization of voluntary eye blinking kinematics. $J R$ Soc Interface 10 (2013).

21. Salvucci, D. \& Goldberg, J. Identifying fixations and saccades in eye-tracking protocols. ETRA '00: Proceedings of the 2000 symposium on Eye tracking research \& applications, 71-78, doi:https://doi.org/10.1145/355017.355028 (2000).

22. Tanaka, M., Yoshida, T. \& Fukushima, K. Latency of saccades during smooth-pursuit eye movement in man - Directional asymmetries. Exp. Brain Res. 121, 92-98 (1998).

23. Engel, K. C., Anderson, J. H. \& Soechting, J. F. Oculomotor tracking in two dimensions. J. Neurophysiol. 81, 1597-1602 (1999).

24. Erkelens, C. J. Coordination of smooth pursuit and saccades. Vision Res. 46, 163-170, doi:10.1016/j.visres.2005.06.027 (2006).

25. Takahashi, M., Uemura, T. \& Fujishiro, T. Quantitative analysis of pursuit eye movements by unidirectional target motion. Arch. Otorhinolaryngol. 238, 225-232, doi:10.1007/BF00453933 (1983).

\section{Tables}

\section{Table 1. Subject characteristics}


SE (D)

Angle of deviation (PD)

ID Age (years) Dominant eye Nondominant eye Near Far Stereoacuity

$(\log \operatorname{arcsec})$

\begin{tabular}{|c|c|c|c|c|c|c|}
\hline S1 & 27 & -0.50 & -0.50 & -4 & -4 & 1.60 \\
\hline S2 & 21 & -1.00 & -0.50 & -2 & -4 & 1.60 \\
\hline S3 & 23 & +0.50 & +0.50 & -4 & -2 & 1.60 \\
\hline S4 & 22 & -0.625 & -0.625 & -6 & -14 & 1.70 \\
\hline S5 & 20 & -4.375 & -2.875 & -6 & -6 & 1.60 \\
\hline S6 & 22 & -0.75 & -0.50 & 0 & 0 & 1.60 \\
\hline S7 & 21 & -0.125 & -0.875 & -6 & -6 & 1.60 \\
\hline S8 & 21 & -6.125 & -5.875 & -8 & -16 & 1.60 \\
\hline S9 & 21 & -3.75 & -3.125 & -2 & -4 & 1.60 \\
\hline S10 & 21 & -3.375 & -3.375 & -8 & -10 & 1.60 \\
\hline S11 & 21 & -7.00 & -7.625 & -6 & -10 & 1.60 \\
\hline S12 & 21 & -4.875 & -3.875 & -10 & -18 & 1.60 \\
\hline $\mathrm{S} 13$ & 21 & -0.375 & -1.125 & -2 & -2 & 1.60 \\
\hline S14 & 21 & -1.25 & -1.125 & -8 & -18 & 1.60 \\
\hline S15 & 21 & -3.25 & -3.25 & -2 & -8 & 1.60 \\
\hline S16 & 21 & -4.50 & -4.75 & -1 & -6 & 1.70 \\
\hline S17 & 21 & -5.25 & -4.75 & 0 & -4 & 1.70 \\
\hline S18 & 20 & -3.875 & -3.125 & -14 & -20 & 1.60 \\
\hline S19 & 22 & -11.5 & -10.125 & -8 & -18 & 1.78 \\
\hline $\mathrm{S} 20$ & 20 & -0.125 & 0.00 & -4 & -10 & 1.60 \\
\hline S21 & 21 & -3.00 & -4.125 & -2 & -4 & 1.60 \\
\hline Mean & 21.4 & -3.23 & -3.09 & -10.9 & -6.3 & 1.62 \\
\hline SD & 1.4 & 3.00 & 2.80 & 8.8 & 5.9 & 0.05 \\
\hline
\end{tabular}

Minus and plus signs in the angle of deviation indicate exodeviation and esodeviation of phoria, respectively. A stereoacuity of $1.60,1.70$, and $1.78 \mathrm{log}$ arcsec is equal to 40,50 , and $60 \mathrm{~s}$, respectively. 
S, subject; SE, spherical equivalent; D, diopter; PD, prism diopter; log arcsec, logarithm of arc second; SD, standard deviation.

Table 2. Accuracy of the classification for each direction in all subjects.

\begin{tabular}{|lll|}
\hline Algorithm & Correct & Incorrect \\
\hline Peak & 184 & 0 \\
\hline Threshold & 88 & 96 \\
\hline
\end{tabular}

A total of 23 healthy subjects participated in this study. The algorithms identified eight directions in each subject. Hence, 184 data points were analyzed. The algorithm of peak fitting-based detection was classified correctly in all directions of target orientation.

peak, peak fitting-based detection; threshold, threshold-based detection.

Table 3. Latencies in all directions.

\begin{tabular}{|c|c|c|c|}
\hline & Latency (ms) & & \\
\hline Direction & LE & $\mathrm{RE}$ & $P$ value \\
\hline Left & $97.10 \pm 83.43$ & $120.29 \pm 89.18$ & 0.56 \\
\hline Right & $104.34 \pm 86.63$ & $101.45 \pm 104.68$ & $>0.99$ \\
\hline Upper left & $142.03 \pm 106.94$ & $172.46 \pm 107.14$ & 0.56 \\
\hline Upper right & $118.84 \pm 94.72$ & $124.64 \pm 95.99$ & $>0.99$ \\
\hline Lower left & $152.17 \pm 108.16$ & $162.32 \pm 122.81$ & $>0.99$ \\
\hline Lower right & $184.06 \pm 113.20$ & $168.12 \pm 108.47$ & $>0.99$ \\
\hline Upper & $100.00 \pm 70.35$ & $120.29 \pm 106.71$ & $>0.99$ \\
\hline Lower & $127.54 \pm 97.26$ & $114.49 \pm 102.41$ & $>0.99$ \\
\hline
\end{tabular}

The error term is the standard deviation.

$m s$, milliseconds; LE, left eye; RE, right eye.

Table 4. Gains in all directions. 
Gain

\begin{tabular}{llll}
\hline Direction & LE & RE & $P$ value \\
\hline Left & $0.957 \pm 0.175$ & $0.97 \pm 0.171$ & $>0.99$ \\
\hline Right & $0.940 \pm 0.153$ & $0.931 \pm 0.142$ & $>0.99$ \\
\hline Upper left & $0.946 \pm 0.122$ & $0.923 \pm 0.147$ & $>0.99$ \\
\hline Upper right & $0.966 \pm 0.203$ & $0.921 \pm 0.149$ & $>0.99$ \\
\hline Lower left & $0.971 \pm 0.186$ & $0.921 \pm 0.113$ & $>0.99$ \\
\hline Lower right & $0.925 \pm 0.156$ & $0.936 \pm 0.113$ & $>0.99$ \\
\hline Upper & $0.860 \pm 0.199$ & $0.904 \pm 0.222$ & $>0.99$ \\
\hline Lower & $0.908 \pm 0.233$ & $0.849 \pm 0.204$ & $>0.99$
\end{tabular}

The error term is the standard deviation.

ms, milliseconds; LE, left eye; RE, right eye.

\section{Figures}

\section{Figure 1}

\section{Setting the relative origin.}

In clinical settings, the target that is presented by the examiner $(A)$ does not always coincide with the center of the scene camera (B). The median of the target location was calculated both horizontally and vertically, respectively, and was defined as the relative origin (C).

\section{Figure 2}

\section{Algorithm of peak fitting-based detection}

The target location was converted to the position vector, and then, the maximum and minimum peaks were detected for $3.0 \mathrm{~s}(\mathrm{~A})$. (B) Data between two minimum peaks, including one maximum peak in the green square of $(A)$. The separated data were decomposed into horizontal (C) and vertical (D) components from the position vector. After excluding $1 \mathrm{~s}$ from both ends of the separated data (black vertical lines in $C$ and $D$ ), the medians of the horizontal and vertical target locations were calculated and plotted (E). (F) Superimposition of the relative origin data, which is the same as Figure 1C of the target. 


\section{Figure 3}

\section{Categorizing each direction.}

The eight horizontal and vertical median locations were ranked from maximum to minimum at left (green ellipse), right (yellow ellipse), upper (blue ellipse), and lower (red ellipse), and then, the top three values in the four directions were grouped (A). Then, the plots that belong to the two groups (upper left, upper right, lower left, and lower right) were identified by combining the horizontal and vertical directions (the red squares in B). Each plot was numbered according to the order of the maximum peak calculated in Figure $2 A$; thus, the fifth waveform is in the upper left.

\section{Figure 4}

\section{Calculating latency.}

The horizontal and vertical target locations and both eye positions were converted to the position vector (A). (A) were fitted with a cubic function and detected at each peak time (B). (C) Each peak time was applied to (A). The latencies of both eyes were defined as the difference between the peak time in both eyes and that in the target location.

\section{Figure 5}

\section{Calculating gain.}

The target location and both eye positions at the peak time were defined as maximum values. The 25th and 75th percentile points of the maximum values in the centrifugal direction were explored (the green horizontal lines). The gains of both eyes were defined as the ratio of the slope of the regression line in both eyes to the slope of the regression line in the target (the black dashed line) between the 25th and 75th percentile points.

\section{Figure 6}

\section{Latencies of the left (A) and right (B) eyes in each direction.}

The latencies in all directions were not significantly different within both eyes ( $A$ and $B$ ). The blue and red boxplots with dots indicate the latency of the left and right eyes in each direction (C). The latencies in all 
directions were not significantly different between the left and right eyes.

\section{Figure 7}

\section{Gains of the left (A) and right (B) eyes in each direction.}

The gains in all directions were not significantly different within both eyes ( $A$ and $B$ ). The blue and red boxplots with dots indicate the latency of the left and right eyes in each direction (C). The gains in all directions were not significantly different between left and right eyes. 